\title{
The impact of adjuvant therapy on survival for node-negative esophageal squamous cell carcinoma: a propensity score- matched analysis
}

\author{
Xuyang Deng ${ }^{1 \#}$, Wenwu $\mathrm{He}^{2 \#}$, Yingchun Jiang ${ }^{2}$, Sijie Deng ${ }^{3}$, Tianqin Mao ${ }^{4}, \mathrm{Xuefeng} \mathrm{Leng}^{2}$, Qiyu $\mathrm{Luo}^{4}$, \\ Kai Zheng ${ }^{1}$, Yongtao Han $^{1,2}$ \\ ${ }^{1}$ School of Medicine, Southwest Medical University, Luzhou, China; ${ }^{2}$ Department of Thoracic Surgery, Sichuan Cancer Hospital \& Research \\ Institute, School of Medicine, University of Electronic Science and Technology of China (UESTC), Chengdu, China; ${ }^{3}$ Department of \\ Endocrinology, People's Hospital of Deyang City, Deyang, China; ${ }^{4}$ School of Medicine, University of Electronic Science and Technology of China \\ (UESTC), Chengdu, China \\ Contributions: (I) Conception and design: W He, X Deng; (II) Administrative support: Y Han, W He; (III) Provision of study materials or patients: \\ Y Jiang, S Deng, T Mao, K Zheng, Q Luo; (IV) Collection and assembly of data: S Deng, T Mao, K Zheng, Q Luo; (V) Data analysis and \\ interpretation: X Leng, X Deng; (VI) Manuscript writing: All authors; (VII) Final approval of manuscript: All authors. \\ \#These authors contributed equally to this work. \\ Correspondence to: Yongtao Han, MD, FRCS. School of Medicine, Southwest Medical University, Luzhou, China; Department of Thoracic Surgery, \\ Sichuan Cancer Hospital \& Research Institute, School of Medicine, University of Electronic Science and Technology of China (UESTC), Chengdu, \\ China. Email: yongtao_han@126.com.
}

Background: At present, the primary treatment of esophageal cancer is surgery-based comprehensive treatment, including adjuvant therapy such as chemotherapy and/or radiotherapy. However, the role of adjuvant therapy for esophageal squamous cell carcinoma (ESCC) with pathologically node-negative (pN0) disease is controversial. This study aimed to evaluate the impact of postoperative adjuvant therapy on survival in patients with pN0 ESCC.

Methods: Patients with ESCC who underwent R0 esophagectomy in the Department of Thoracic Surgery of Sichuan Cancer Hospital from January 2008 to December 2013 were enrolled. Patients were divided into two groups: a surgery alone (Group S) group or a surgery + adjuvant therapy (Group S + A) group. The primary outcomes were overall survival (OS) and disease-free survival (DFS), and every consecutive case was followed up until death or the last follow-up.

Results: A total of 387 patients with ESCC patients who had pN0 were enrolled in the study. After propensity score matching (PSM), each group consisted of 150 patients. In the overall cohort, the 5-year OS (75.6\% vs. $69.7 \%$; $\mathrm{P}=0.004)$ and 5 -year DFS (64.9\% vs. $48.2 \% ; \mathrm{P}=0.003)$ rates were higher in Group $\mathrm{S}+\mathrm{A}$ than in Group S. In the matched samples, the same outcomes were observed (5-year OS: $75.6 \%$ vs. 69.7\%, $\mathrm{P}=0.026 ; 5$-year DFS: $67.6 \%$ vs. $69.6 \%, \mathrm{P}=0.036)$. Multivariate regression analysis indicated that postoperative chemotherapy was associated with longer OS [hazard ratio (HR): 0.622, 95\% confidence interval (CI): 0.416-0.928; P=0.02] and DFS (HR: 0.571, 95\% CI: 0.390-0.836; P=0.004); in contrast, T3 stage tumors (HR: 1.953, 95\% CI: 1.238-3.082; P=0.004) and <15 lymph node dissections (HR: $1.81 ; 95 \%$ CI: $1.238-2.648 ; \mathrm{P}=0.002$ ) were found to be independent risk factors for pN0 ESCC.

Conclusions: Adjuvant therapy, especially chemotherapy, prolonged OS and DFS for patients with ESCC who had pN0 disease. Fewer lymph node dissections and T3 stage tumors were independent risk factors for OS and DFS.

Keywords: Adjuvant therapy; esophageal squamous cell carcinoma (ESCC); lymph node-negative

\footnotetext{
$\wedge$ ORCID: 0000-0002-6807-4239.
} 
Submitted May 06, 2021. Accepted for publication Jun 16, 2021.

doi: 10.21037/atm-21-2539

View this article at: https://dx.doi.org/10.21037/atm-21-2539

\section{Introduction}

The prognosis of esophageal cancer is poor, with the 5 -year overall survival (OS) being lower than $40 \%(1,2)$. The most common pathological type of esophageal cancer in China is esophageal squamous cell carcinoma (ESCC) (3), and the dominant treatment for esophageal cancer is comprehensive treatment based on surgery. The National Comprehensive Cancer Network (NCCN) guidelines suggest that pathologically node-negative $(\mathrm{pN} 0)$ patients with R0 resection require only follow-up and not adjuvant therapy. However, in clinical practice, postoperative adjuvant therapies are selectively applied to patients with pN0 ESCC. Indeed, for some time now, postoperative adjuvant therapy has been performed on the basis of doctors; judgment, and thus adjuvant therapy strategies for ESCC are diverse. Some studies have discussed the relationship between postoperative adjuvant therapy and the survival status of patients with pN0 ESCC, but the conclusions remain controversial (4-9); overall the number of studies focusing on this issue is insufficient, and so the value of postoperative adjuvant treatment for pN0 ESCC remains unclear. Previous related studies have mostly concentrated on the relationship between the prognosis of patients with lymph node-positive esophageal cancer and adjuvant therapy. This study focuses on lymph nodenegative ESCC. Different treatment methods are included in the analysis. The case data is closer to the current time, the follow-up survival data is complete, and the application of propensity-score matching eliminates bias as much as possible. Consequently, exploring therapies to improve the prognosis of patients with pN0 ESCC might be considerably beneficially, and thus this study was performed to evaluate the value of postoperative adjuvant therapies for patients with pN0 ESCC. We present the following article in accordance with the STROBE reporting checklist (available at https://dx.doi. org/10.21037/atm-21-2539).

\section{Methods}

\section{Study population}

The initial data consisted of 387 patients in total, and patients who underwent radical esophagectomy in Sichuan Cancer Hospital from January 2008 to December 2013 were involved. Inclusion criteria were as follows: (I) patients with pN0 ESCC according to the postoperative pathology reports; (II) no neoadjuvant therapy or molecular targeted therapy before esophagectomy; and (III) Eastern Cooperative Oncology Group (ECOG) performance status of $0-2$. The exclusion criteria were as follows: (I) pathologically positive margin; (II) death related to the surgery in 3 months; (III) diagnosis of any other malignancy simultaneously; and (IV) refusal of followup. Data collection and the last follow-up were completed in December 2013, and the data were collected from telephone follow-ups, the hospital information center, and local household registration management departments. All patients were divided among two groups: a surgery alone (Group S) group or a surgery + adjuvant therapy (Group S + A) group, the flow chart is shown in Figure 1. All procedures performed in this study involving human participants were in accordance with the Declaration of Helsinki (as revised in 2013). The study was approved by the Ethics Committee of Sichuan Cancer Hospital, and all patients were informed of the study and consented to participate.

\section{Surgery}

All patients underwent radical esophagectomy and two (mediastinal and perigastric) or three (cervical, mediastinal, and perigastric) fields of regional lymphadenectomy. Surgical methods included right thoracotomy with or without thoracoscopy but with transhiatal esophagectomy. The anastomotic site was related to tumor location. In general, cervical anastomosis was performed in patients with upper esophageal tumors. A supra-aortic arch esophagogastric anastomosis was performed for patients with middle or lower esophageal lesions. Reconstruction of the alimentary tract was performed using the stomach or jejunum after esophageal resection for carcinoma.

\section{Adjuvant therapy}

The general postoperative adjuvant treatment procedure at our institution is pathological T1a-3N1-3 and T4a-4bNx, 


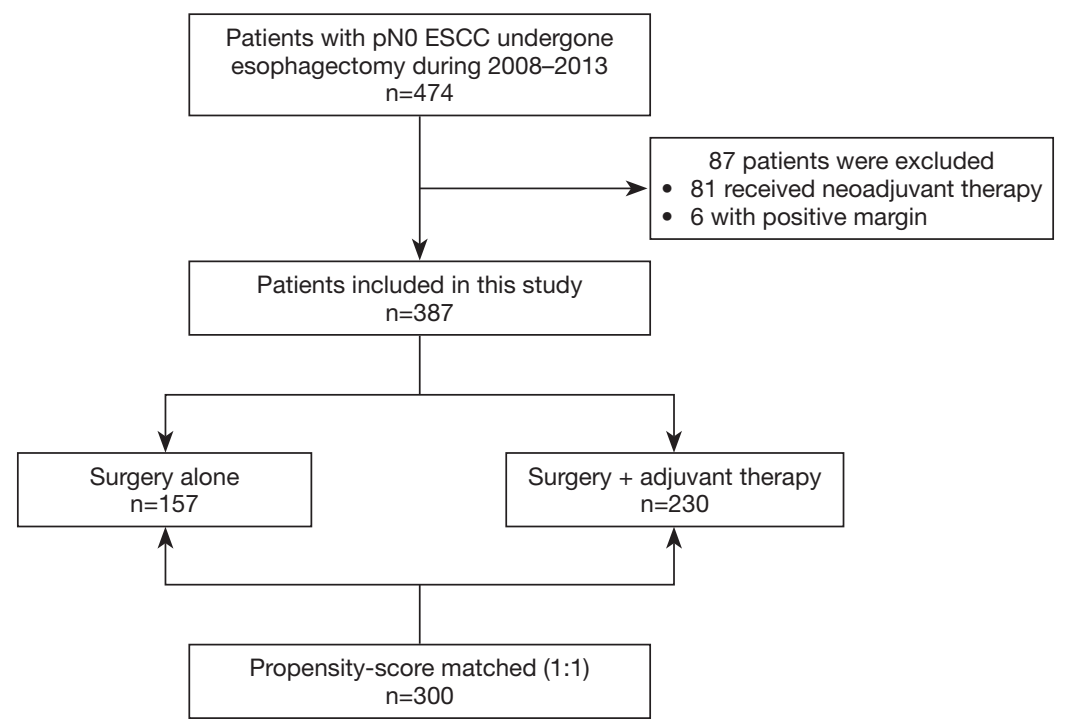

Figure 1 Flow chart of the study. pN0, pathologically node-negative; ESCC, esophageal squamous cell carcinoma.

R1 resection, and nerve or vascular invasion. However, when we provide postoperative adjuvant treatment for pN0 patients, the surgeon not only takes into account the patient's pathological results and intraoperative conditions but also the patient's wishes. The following adjuvant chemotherapy regimens were applied: paclitaxel (a dose of $135-175 \mathrm{mg} / \mathrm{m}^{2}$ ) combined with cisplatin (a dose of $80-100 \mathrm{mg} / \mathrm{m}^{2}$ ) or carboplatin (a dose of $300 \mathrm{mg} / \mathrm{m}^{2}$ ) for 2-4 courses; each course of chemotherapy lasted 28 days (4 weeks). A subsequent or concurrent radiotherapy course of a total dose of 50-60 Gy in 25-30 fractions 5 days a week was given $2-4$ weeks after or during chemotherapy administration. According to the results of the computed tomography (CT) scan, the radiation field was delineated using conformal intensity-modulated radiation therapy, including mediastinal, bilateral supraclavicular, and epigastric radiation.

\section{Outcomes}

The primary outcomes were 5-year OS and disease-free survival (DFS) after the operation. OS was assessed as the interval between the date of surgery and the date of death from any cause, loss to follow-up, or last follow-up. DFS was assessed as the interval between the date of surgery and the date of first recurrence, death from any cause, loss to follow-up, or last follow-up. Recurrence was defined as local regional recurrence, lymph node metastasis, or distant metastasis.

\section{Statistical analyses}

Survival data were analyzed by the Kaplan-Meier method, while the log-rank method was applied to compare survival curves between groups. Univariate and multivariate analyses of OS and DFS were conducted by Cox regression models with stepwise selection. Fisher's exact test or chi-square test for categorical variables and Student's $t$-test or WilcoxonMann-Whitney test for continuous variables were applied as appropriate. To balance covariates (10), 1:1 propensity score matching (PSM) was performed, with age, gender, tumor location, differentiation, and lymph node dissection being the covariates considered, and the matching tolerance set to 0.1 . A two-tailed $\mathrm{P}$ value $<0.05$ was defined as statistically significant. Statistical analysis was performed using the SPSS 26.0 (IBM Corp., Armonk, NY, USA).

\section{Results}

\section{Patients' demographics}

From 2008 to 2013 in a total of 387 patients at Sichuan Cancer Hospital with postoperative pathology of $\mathrm{pN} 0$ ESCC were included in the overall cohort (Table 1). Of the total cohort, 157 patients were in Group S, and 230 patients were in Group S + A. In Group S + A, 196 patients received postoperative chemotherapy and 34 patients received chemoradiotherapy. The covariates of age, sex, and tumor location were unbalanced in the two groups. After PSM, 87 patients were excluded, and the matched cohort included 
Table 1 Patients' characteristics before and after PSM

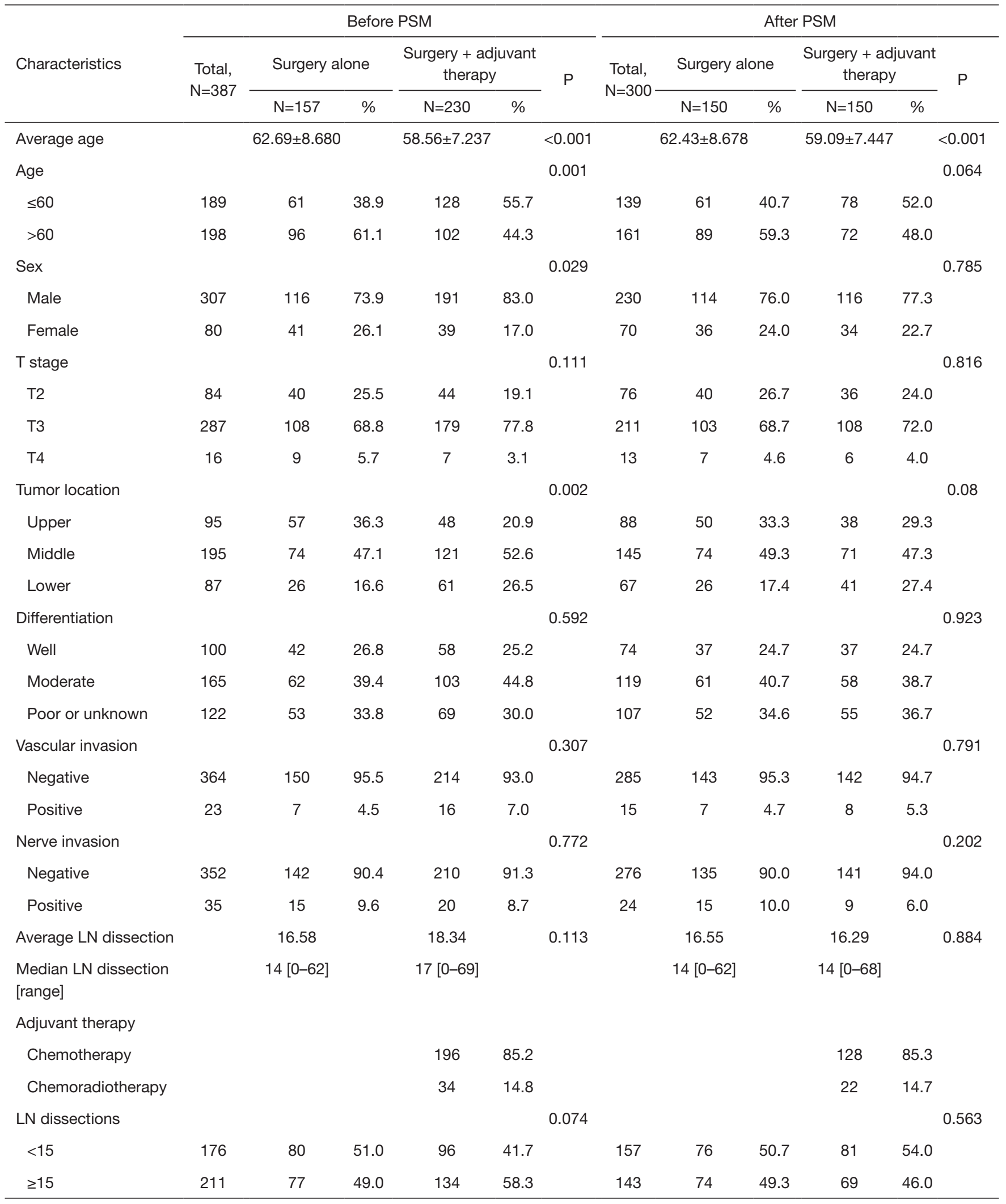

PSM, propensity score matching; LN, lymph node. 


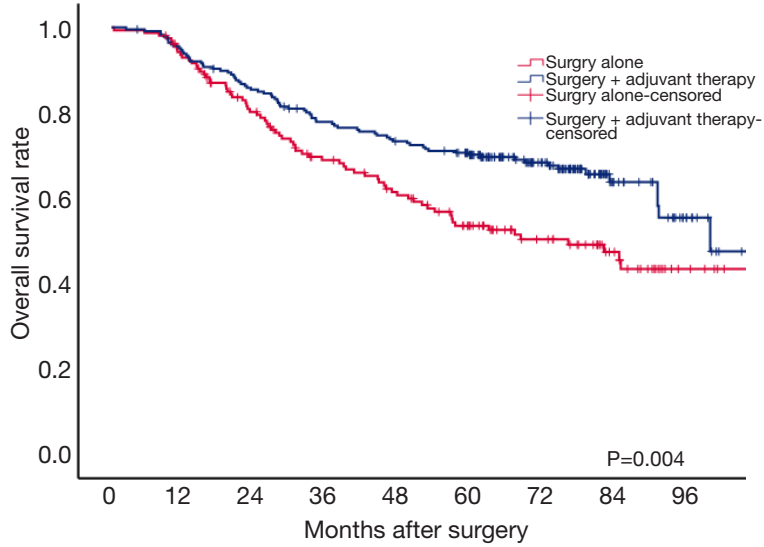

Figure 2 OS between surgery alone and surgery + adjuvant therapy groups before PSM. OS, overall survival; PSM, propensity score matching.

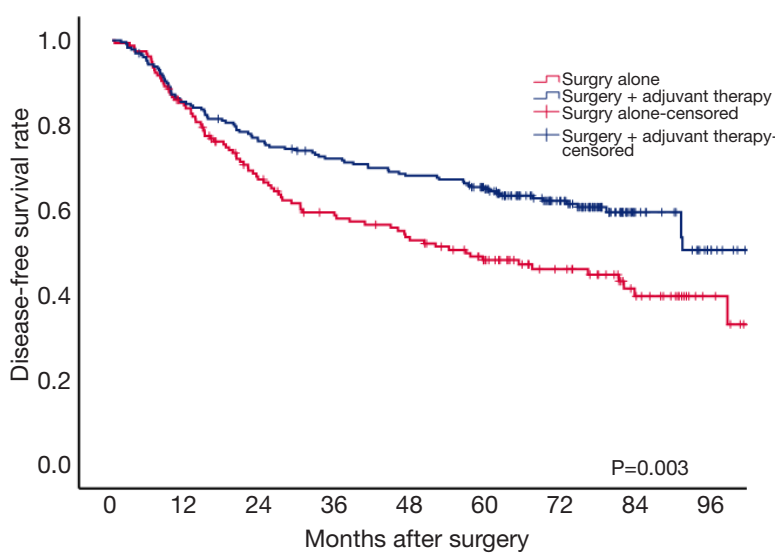

Figure 3 DFS between surgery alone and surgery + adjuvant therapy groups before PSM. DFS, disease-free survival; PSM, propensity score matching.

150 patients in Group S and 150 patients in Group S + A (Table 1).

\section{OS and DFS}

Across the whole study population, the median followup was 75.60 [95\% confidence interval (CI): $73.17-$ 78.03] months. The median follow-up was 76.27 (95\% CI: 65.44-87.09) months in Group S and 75.6 (95\% CI: 73.89-77.31) months in Group S + A. In the overall cohort, 74 patients died in Group S, and 79 in Group S + A; the 3 - and 5-year OS rates were $69 \%$ and $53.6 \%$ in Group S, respectively, which were significantly lower than the OS

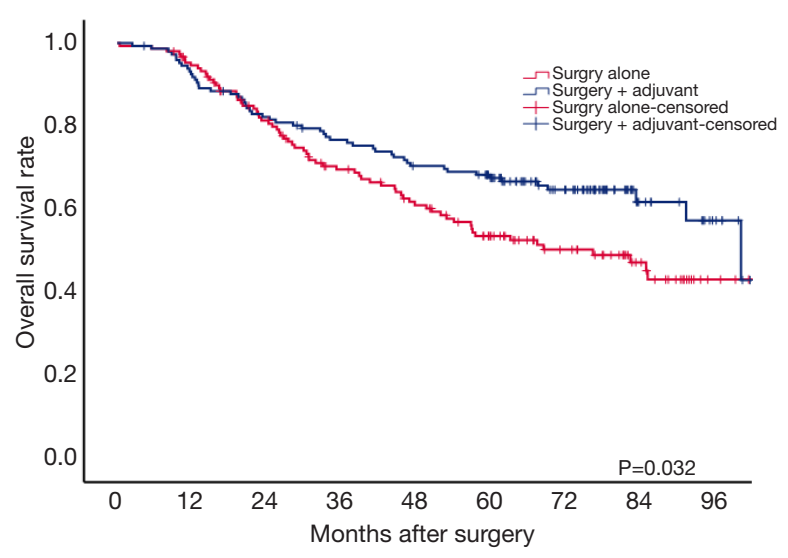

Figure 4 OS between surgery alone and surgery + adjuvant therapy groups after PSM. OS, overall survival; PSM, propensity score matching.

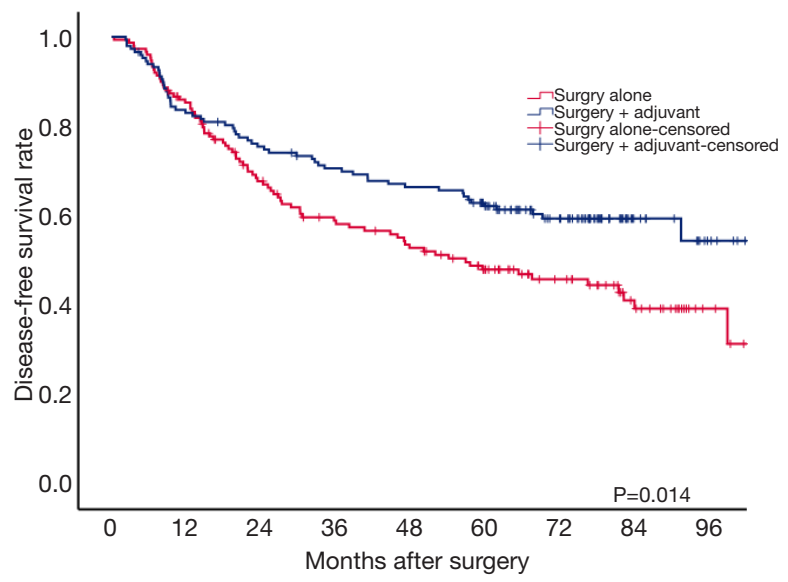

Figure 5 DFS between surgery alone and surgery + adjuvant therapy groups after PSM. DFS, disease-free survival; PSM, propensity score matching.

rates in Group S + A (3-year: 77.9\%; 5-year: 70.2\%; logrank $\chi^{2}=8.222 ; \mathrm{P}=0.004 ;$ Figure 2 ). The 3 - and 5 -year DFS rates in Group S were $58 \%$ and $48.2 \%$, respectively, which were lower than the DFS rates in Group S + A (3-year: 72.2\%; 5-year: 64.9\%; log-rank $\chi^{2}=8.684 ; \mathrm{P}=0.003$; Figure 3).

In the matched cohort, the 3 - and 5 -year OS rates were $69.6 \%$ and $53.6 \%$ in Group S, respectively, which were significantly lower than the OS rates in Group S + A (3-year: 76.7\%; 5-year: 67.6\%; log-rank $\chi^{2}=4.614 ; \mathrm{P}=0.032$; Figure 4). In Group S, the 3- and 5-year DFS rates were $58.2 \%$ and $48 \%$, respectively, which were significantly lower than the DFS rates in Group S + A (3-year: 70.6\%; 5-year: $62.6 \%$; log-rank $\chi^{2}=6.083 ; \mathrm{P}=0.014 ;$ Figure 5). In 

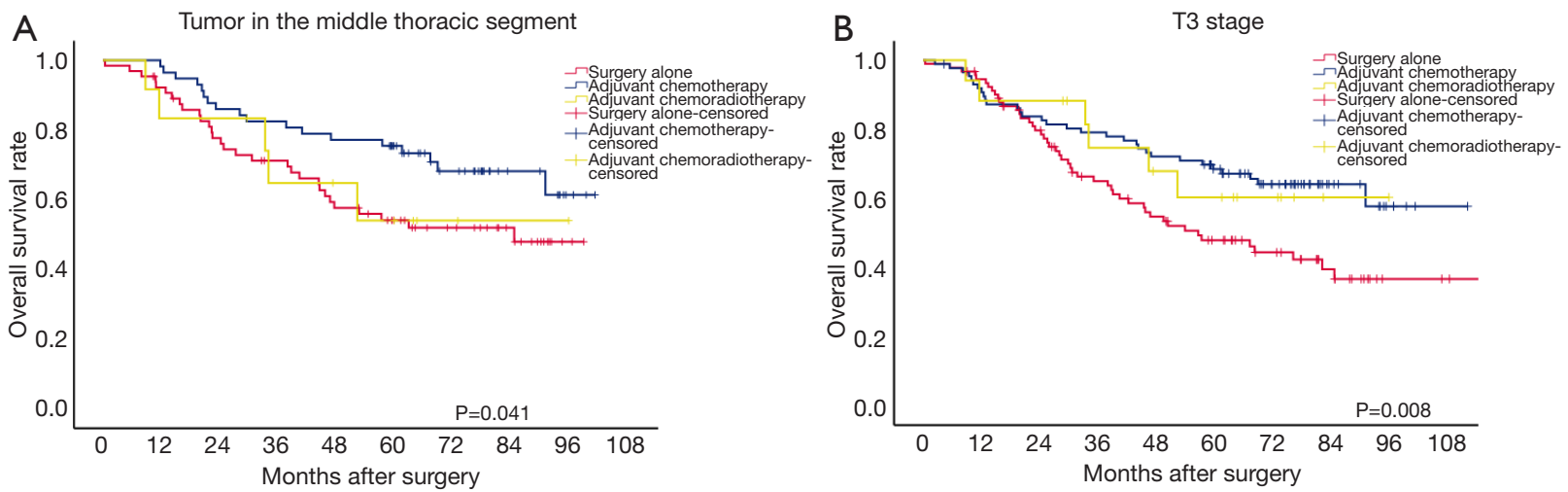

Figure 6 Surgery + adjuvant chemotherapy improved OS in patients with tumor in the middle thoracic segment $(\mathrm{P}=0.041, \mathrm{~A})$ and $\mathrm{T} 3$ stage $(\mathrm{P}=0.008, \mathrm{~B})$. OS, overall survival.

hierarchical analysis, postoperative chemotherapy prolonged OS in patients with tumors in the middle thoracic segment $(\mathrm{P}=0.041)$ and $\mathrm{T} 3$ stage tumors $(\mathrm{P}=0.008)$ compared with surgery alone (Figure 6), but differences were not found between postoperative chemoradiotherapy and surgery alone or postoperative chemotherapy. Compared with surgery alone, postoperative chemotherapy improved DFS in patients with moderate differentiation $(\mathrm{P}=0.023)$, tumors in the middle thoracic segment $(\mathrm{P}=0.028)$, $\mathrm{T} 3$ stage tumors $(\mathrm{P}=0.004)$, and $<15$ lymph node dissections $(\mathrm{P}=0.038$; Figure 7), and no significant differences were observed between postoperative and other therapies (surgery alone and postoperative chemotherapy).

\section{Multivariate analysis of DFS}

Multivariate analysis was performed in a matched cohort. Time, sex, age, T stage, tumor location, vascular invasion, nerve invasion, histologic differentiation, type of treatment, and lymph node dissection were included in the Cox regression analysis to evaluate the prognostic factors for DFS through calculation of hazard ratios (HRs) and their 95\% CIs (Table 2). The results showed that postoperative adjuvant chemotherapy was an independent protective factor (HR: 0.571; 95\% CI: 0.390-0.836; $\mathrm{P}=0.004$; Figure 8), and T3 stage tumors (HR: 1.953; 95\% CI: 1.238-3.082; $\mathrm{P}=0.004$ ) and $<15$ lymph node dissections (HR: 1.81; 95\% CI: $1.238-$ 2.648; $\mathrm{P}=0.002$ ) were independent risk factors for prognosis.

\section{Discussion}

We assessed the value of postoperative adjuvant therapy in patients with pN0 ESCC. The results suggested that postoperative adjuvant therapy prolonged OS and DFS in patients with pN0 ESCC, which is converse to results reported for esophageal adenocarcinoma (11). Univariate analysis of the two groups in the PSM cohort revealed that T3 stage, moderate and poor differentiation, and $<15$ lymph node dissections were risk factors for prognosis. Moreover, Cox multivariate regression analysis indicated that postoperative adjuvant chemotherapy was an independent protective factor, and $\mathrm{T} 3$ tumors and $<15$ lymph node dissections were independent risk factors for prognosis. We chose DFS as the primary outcome in this study because, after recurrence, patients could be treated with any therapy considered useful. In the comparison of postoperative adjuvant therapy and surgery alone, Yang et al. reported that postoperative modified conformal radiotherapy was associated with improvements in both OS and DFS in patients with pT3N0M0 ESCC (12). Another study indicated that radiotherapy and postoperative chemoradiation did not significantly improve DFS and OS $(\mathrm{P}=0.692 ; \mathrm{P}=0.368)(13)$, but the study focused on patients with ESCC with positive lymph node(s). Chen et al. examined 426 patients and found that postoperative adjuvant chemotherapy did not improve OS, regardless of lymph node metastasis (14), but the authors pointed out the problem of inconsistent chemotherapy regimens. We also found that postoperative adjuvant treatment affected the patient's nutritional status, which in turn affected immune function. A meta-analysis of surgery + adjuvant chemotherapy and surgery alone for patients with resectable thoracic ESCC revealed that postoperative chemotherapy did not 

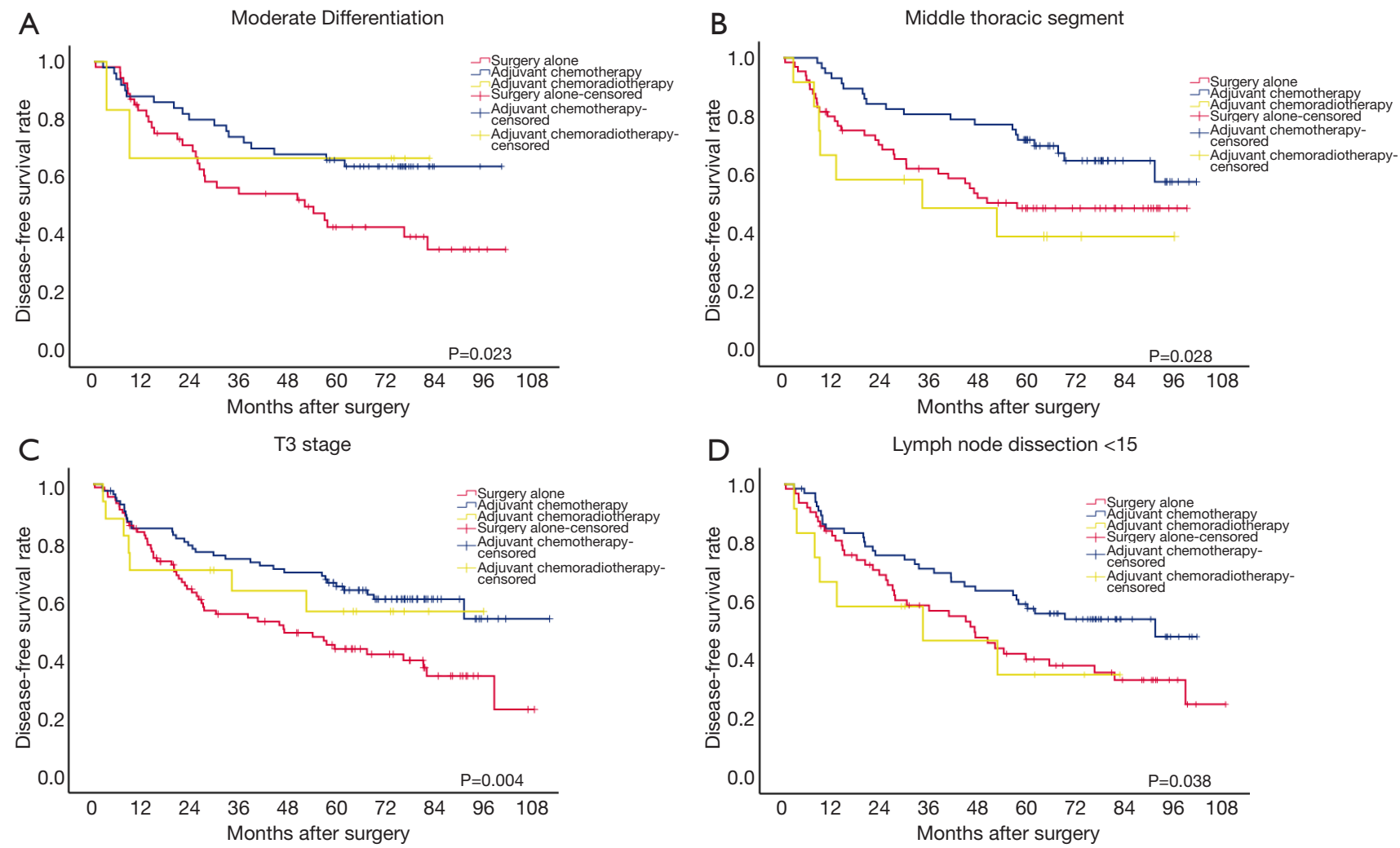

Figure 7 Surgery + adjuvant chemotherapy improved DFS in patients with moderate differentiation $(\mathrm{P}=0.023, \mathrm{~A})$, tumor in the middle thoracic segment $(\mathrm{P}=0.028, \mathrm{~B})$, T3 stage tumor $(\mathrm{P}=0.004, \mathrm{C})$, and $<15$ lymph node dissections $(\mathrm{P}=0.038, \mathrm{D})$. DFS, disease-free survival.

improve the patient's OS but did extend 1-year DFS (15). A Japanese multicenter randomized controlled trial (JCOG9204) found that postoperative adjuvant therapy alone prolonged 5-year DFS compared with the surgery alone $(55 \%$ vs. $45 \% ; \mathrm{P}=0.037)$ in patients with $\mathrm{pN} 0$ ESCC (5). A more remarkable difference was found in patients with pN1 ESCC $(\mathrm{P}=0.041)$. The regimen of JCOG9204 was cisplatin and fluorouracil, but the treatment applied to patients was complicated due to individual discrepancies after recurrence. Some study results have supported the JCOG9204 conclusion and indicated that patients with stage III-IV disease can obtain a long-term survival benefit from postoperative chemotherapy $(8,9)$, but this may be a dependent on invasion depth. In a study focusing on patients with stage III disease, Yang et al. found that adjuvant therapy improved the OS and DFS of patients with ESCC (16). Further analysis indicated that lesions of the middle thorax and well-differentiated tumors had a longer OS $(\mathrm{P}<0.05)$. Additionally, Zhang et al. observed that concurrent chemoradiotherapy improved the OS of patients with resectable stage III-IV ESCC (17), but did not examine N0 patients. In Zhang et al.'s analysis of patients with pT3N0M0 thoracic ESCC with surgery alone versus those with postoperative adjuvant therapy (chemotherapy/ radiotherapy/radiochemotherapy), the results suggested that postoperative adjuvant therapy improves the DFS of these patients; furthermore, postoperative radiotherapy was found to improve DFS in the mid-segment, moderately differentiated, and $<15$ lymph node dissections subgroups, while multivariate analysis demonstrated that adjuvant radiochemotherapy and female sex can improve survival (7).

There were several limitations to our study that should be noted. First, our study had a retrospective design. Although the PSM procedure can avoid potential biases, unlike in randomized controlled trials, these biases still may not be completely eliminated. Second, this was a single-institution study, and the rate of administration of chemoradiation $(n=22)$ in our cohort was much lower than that of surgery alone $(n=150)$ and postoperative chemotherapy $(n=122)$. Nonetheless, our findings may provide some guidance for 
Table 2 Univariate and multivariate Cox analysis of patients after PSM

\begin{tabular}{|c|c|c|c|c|c|c|}
\hline Variables & \multicolumn{3}{|c|}{ Univariate } & \multicolumn{3}{|c|}{ Multivariate } \\
\hline \multicolumn{7}{|l|}{ Age } \\
\hline$<60$ & 1 & & & 1 & & \\
\hline$\geq 60$ & 0.931 & $0.667-1.299$ & 0.675 & 0.925 & $0.643-1.330$ & 0.673 \\
\hline Male & 1 & & & 1 & & \\
\hline Female & 0.855 & $0.570-1.282$ & 0.448 & 0.925 & $0.583-1.467$ & 0.74 \\
\hline Tumor location & & & 0.413 & & & \\
\hline Upper & 1 & & & & & \\
\hline T stage & & & 0.055 & & & 0.015 \\
\hline $\mathrm{T} 2$ & 1 & & & 1 & & \\
\hline T3 & 1.675 & $1.098-2.555$ & 0.017 & 1.953 & $1.238-3.082$ & 0.004 \\
\hline $\mathrm{T} 4$ & 1.328 & $0.511-3.451$ & 0.561 & 1.444 & $0.470-4.441$ & 0.521 \\
\hline Differentiation & & & 0.029 & & & 0.414 \\
\hline Well & 1 & & & 1 & & \\
\hline Moderate & 1.761 & $1.094-2.832$ & 0.02 & 1.272 & $0.805-2.012$ & 0.303 \\
\hline Poor or unknown & 1.867 & $1.157-3.014$ & 0.011 & 1.466 & $0.880-2.443$ & 0.142 \\
\hline Positive & 1.153 & $0.586-2.267$ & 0.68 & & & \\
\hline Treatment & & & 0.15 & & & 0.015 \\
\hline Surgery alone & 1 & & & 1 & & \\
\hline Surgery + chemotherapy & 0.694 & $0.480-1.004$ & 0.052 & 0.571 & $0.390-0.836$ & 0.004 \\
\hline Surgery + chemoradiotherapy & 0.913 & $0.438-1.902$ & 0.808 & 0.67 & $0.310-1.450$ & 0.309 \\
\hline \multicolumn{7}{|l|}{ LN dissections } \\
\hline$\geq 15$ & 1 & & & 1 & & \\
\hline$<15$ & 1.935 & $1.363-2.748$ & $<0.001$ & 1.81 & $1.238-2.648$ & 0.002 \\
\hline
\end{tabular}

PSM, propensity score matching; LN, lymph node; HR, hazard ratio; Cl, confidence interval. 


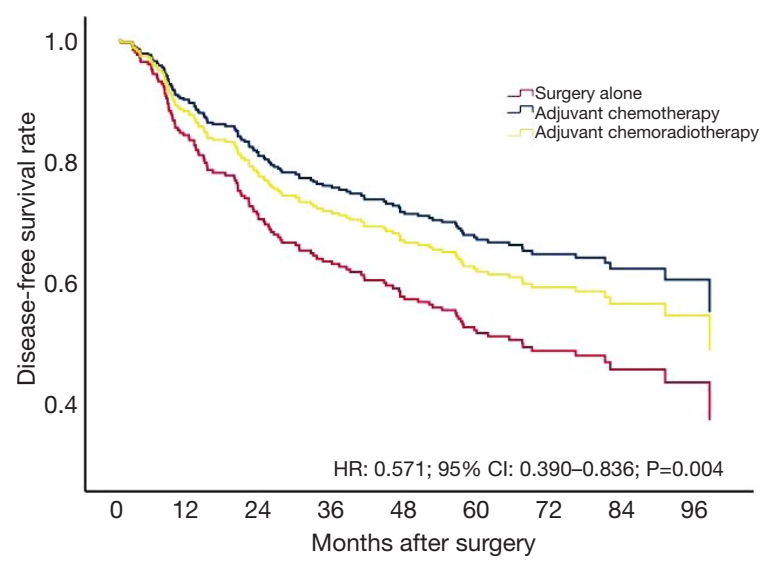

Figure 8 Multivariate analysis of postoperative treatment in the matched cohort across Cox regression. HR, hazard ratio; CI, confidence interval.

clinical work, but more cases and studies are needed to draw more convincing conclusions.

\section{Conclusions}

Postoperative chemotherapy significantly improved the OS and PFS of patients with thoracic pNO ESCC. A future multicenter, randomized controlled clinical trial is warranted to confirm our findings.

\section{Acknowledgments}

The authors also appreciate the academic support from AME Esophageal Cancer Collaborative Group.

Funding: This work was supported by Bethune Charitable Foundation (HZB-20190528-19) and Nanchong City School Science and Technology Strategic Cooperation Project (NSMC20170209).

\section{Footnote}

Reporting Checklist: The authors have completed the STROBE reporting checklist. Available at https://dx.doi. org/10.21037/atm-21-2539

Data Sharing Statement: Available at https://dx.doi. org/10.21037/atm-21-2539

Conflicts of Interest: All authors have completed the ICMJE uniform disclosure form (available at https://dx.doi. org/10.21037/atm-21-2539). The authors have no conflicts of interest to declare.

Ethical Statement: The authors are accountable for all aspects of the work in ensuring that questions related to the accuracy or integrity of any part of the work are appropriately investigated and resolved. All procedures performed in this study involving human participants were in accordance with the Declaration of Helsinki (as revised in 2013). The study was approved by the Ethics Committee of Sichuan Cancer Hospital. Informed consent was taken from all the patients.

Open Access Statement: This is an Open Access article distributed in accordance with the Creative Commons Attribution-NonCommercial-NoDerivs 4.0 International License (CC BY-NC-ND 4.0), which permits the noncommercial replication and distribution of the article with the strict proviso that no changes or edits are made and the original work is properly cited (including links to both the formal publication through the relevant DOI and the license). See: https://creativecommons.org/licenses/by-ncnd/4.0/.

\section{References}

1. Rice TW, Rusch VW, Apperson-Hansen C, et al. Worldwide esophageal cancer collaboration. Dis Esophagus 2009;22:1-8.

2. Allum WH, Stenning SP, Bancewicz J, et al. Long-term results of a randomized trial of surgery with or without preoperative chemotherapy in esophageal cancer. J Clin Oncol 2009;27:5062-7.

3. Wheeler JB, Reed CE. Epidemiology of esophageal cancer. Surg Clin North Am 2012;92:1077-87.

4. Kang J, Chang JY, Sun X, et al. Role of postoperative concurrent chemoradiotherapy for esophageal carcinoma: a meta-analysis of 2165 patients. J Cancer 2018;9:584-93.

5. Ando N, Iizuka T, Ide H, et al. Surgery plus chemotherapy compared with surgery alone for localized squamous cell carcinoma of the thoracic esophagus: a Japan Clinical Oncology Group Study--JCOG9204. J Clin Oncol 2003;21:4592-6.

6. Zhang J, Chen HQ, Zhang YW, et al. Adjuvant chemotherapy in oesophageal cancer: a meta-analysis and experience from the Shanghai Cancer Hospital. J Int Med Res 2008;36:875-82.

7. Zhang X, Yang Y, Sun Y, et al. Adjuvant therapy for 
pathological T3N0M0 esophageal squamous cell carcinoma. J Thorac Dis 2019;11:2512-22.

8. Zhang J, Zhang YW, Chen ZW, et al. Adjuvant chemotherapy of cisplatin, 5-fluorouracil and leucovorin for complete resectable esophageal cancer: a casematched cohort study in east China. Dis Esophagus 2008;21:207-13.

9. Zhu S, Su J, Li J, et al. Survival study of adjuvant radiotherapy and chemotherapy after definitive surgery of locally advanced esophageal carcinoma. International Journal of Radiation Oncology $\bullet$ Biology $\bullet$ Physics 2011;81:S323.

10. da Costa BR, Gahl B, Jüni P. Tools \& techniques-statistics: propensity score techniques. EuroIntervention 2014;10:761-7.

11. Rucker AJ, Raman V, Jawitz OK, et al. The impact of adjuvant therapy on survival after esophagectomy for node-negative esophageal adenocarcinoma. Ann Surg 2020. [Epub ahead of print]. doi: 10.1097/ SLA.0000000000003886.

12. Yang J, Zhang W, Xiao Z, et al. The impact of postoperative conformal radiotherapy after radical surgery on survival and recurrence in pathologic T3N0M0 esophageal carcinoma: a propensity score-matched

Cite this article as: Deng X, He W, Jiang Y, Deng S, Mao T, Leng X, Luo Q, Zheng K, Han Y. The impact of adjuvant therapy on survival for node-negative esophageal squamous cell carcinoma: a propensity score-matched analysis. Ann Transl Med 2021;9(12):998. doi: 10.21037/atm-21-2539 analysis. J Thorac Oncol 2017;12:1143-51.

13. Yu $\mathrm{S}, \mathrm{Zhang} \mathrm{W}, \mathrm{Ni} \mathrm{W}$, et al. A propensity-score matching analysis comparing long-term survival of surgery alone and postoperative treatment for patients in node positive or stage III esophageal squamous cell carcinoma after R0 esophagectomy. Radiother Oncol 2019;140:159-66.

14. Chen H, Wu Z, Chen J, et al. Postoperative adjuvant therapy for resectable thoracic esophageal squamous cell carcinoma: a retrospective analysis of 426 cases. Med Oncol 2015;32:417.

15. Zhang SS, Yang H, Xie X, et al. Adjuvant chemotherapy versus surgery alone for esophageal squamous cell carcinoma: a meta-analysis of randomized controlled trials and nonrandomized studies. Dis Esophagus 2014;27:574-84.

16. Yang Q, Wang YX, He M, et al. Factors affecting on longtime survival in patients with stage III thoracic esophageal carcinoma after esophagectomy. Zhonghua Zhong Liu Za Zhi 2016;38:530-7.

17. Zhang Z, Xu L, Di X, et al. A retrospective study of postoperative radiotherapy for locally advanced esophageal squamous cell carcinoma. Ann Palliat Med 2019;8:708-16.

(English Language Editor: J. Gray) 